Volume 2, Issue 2, April-June 2017, Pages: 169, DOI: http://dx.doi.org/10.19082/ah169

\title{
DATA QUALITY AT THE NORTHEASTERN IRANIAN LIVER TRANSPLANT REGISTRY: AN OVERVIEW OF CONSISTENCY, SPARSENESS, AND TIMELINESS
}

\author{
Tohidinezhad $\mathrm{F}^{1}$, Aliakbarian $\mathrm{M}^{2}$, Akhavan Rezayat $\mathrm{K}^{2}$, Hosseini $\mathrm{MR}^{3}$, Eslami $\mathrm{S}^{4}$
}

1: Student Research Committee, Department of Medical Informatics, Faculty of Medicine, Mashhad University of Medical Sciences, Mashhad, Iran

2: Surgical Oncology Research Center, Mashhad University of Medical Sciences, Mashhad, Iran

3: Department of Internal Medicine, Faculty of Medicine, Mashhad University of Medical Sciences, Mashhad, Iran

4: Pharmaceutical Research Center, Mashhad University of Medical Sciences, Mashhad, Iran

\section{Correspondence:}

Saeid Eslami, Pharmaceutical Research Center, Mashhad University of Medical Sciences, Mashhad, Iran. Tel/Fax: 0098-5138827048, E-mail: EslamiS@mums.ac.ir.

\section{TYPE OF ARTICLE: CONFERENCE ABSTRACT}

\begin{abstract}
Introduction: Due to the prevalence of viral and non-viral hepatic disorders, multiplicity of factors involved in clinical decisions, the need for long-term surveillance of transplant recipients, and continuous prioritization of patients on the waiting list, employing a registry system is considered as a contributory factor to the effective delivery of clinical and scientific objectives. This study is directed to quantify the quality of the first bulk of data entered into our province-wide electronic web-based liver transplant registry system.

Methods: Patients diagnosed with cirrhosis or underwent liver transplant were subjected to this registry from 2013 to 2016. Five and seven inter-record consistency criteria were defined to assess gender, date, and etiological consistency of pre- and post-operation data, respectively and 42 socio-demographic, clinical history, laboratory, imaging, and consultation variables were selected randomly to represent the sparseness of the database. The timeliness was interpreted as the median time from diagnosis to complete registration for each particular patient.

Results: A total of 540 patients accounting for 124 liver transplant recipients and 416 cirrhotic patients were registered during the initial four years. Data consistency was in the range of $89-100 \%$ and sparseness varied between $0.2-20.9 \%$. The median time between diagnosis and registration was 21 days, and this trend appears to be stable over time.

Conclusion: The preliminary appraisal of the database represents the acceptable level of data quality. Embedding required, validation, and consistency check rules following auto-completion feature within structured forms have facilitated and accelerated the data entry process. It is noteworthy that the coding and classification systems follow international standards, and this database is linkable to any national database. We propose to offer the provided database as a rich repository of data to researchers aiming to facilitate data collection process and improve future studies with regards to qualitative and quantitative scientific measures.
\end{abstract}

KEYWORDS: Computerized disease-specific registry, Liver transplantation, Data quality indices

\section{Abstracts of First National Congress of Medical Informatics, Mashhad, Iran, February 2017}

(C) 2017 The Authors. This is an open access article under the terms of the Creative Commons Attribution-NonCommercialNoDerivs License, which permits use and distribution in any medium, provided the original work is properly cited, the use is non-commercial and no modifications or adaptations are made. 